\title{
Less invasive stabilising system plate versus retrograde intramedullary nail for osteoporotic distal femoral fracture
}

\author{
Bhavuk Garg • Rajesh Malhotra • Vijay Kumar • \\ P. P. Kotwal
}

Received: 3 August 2010/Accepted: 28 September 2010/Published online: 14 October 2010

(C) Springer-Verlag 2010

We read with great interest the article "Less invasive stabilizing system plate versus retrograde intramedullary nail for osteoporotic distal femoral fracture" published in May 2010 issue of this esteemed journal. We would like to congratulate the authors for this good study and subject, which really has always a question mark in terms of good outcome. However, we have following concerns:

1. The $25 \%$ patients in LISS group (6 out of 24 patients) and $44 \%$ (4 out of 9 patients) had ipsilateral total knee replacement in situ. They were all classified according to AO classification. They should be considered separate as AO classification does not apply to periprosthetic fractures and it is almost impossible to classify them correctly according to AO classification, even with the help of CT, due to the presence of metallic artefacts. Also, the AO group subclassification is not comparable.

2. The nail group had low patient numbers, making accurate comparison difficult. The authors also did not note if the same design of femoral nail was used in each patient, which may decrease the internal validity of the paper. Currently, there are numerous femoral nails in production including the distal femoral nail (DFN-Synthes, Switzerland which provides very good fixation as well as more fixation options than some other models.'

3. The authors have mentioned that all fractures were low velocity trauma; however, one open fracture has been mentioned in LISS group. This needs to be clarified.

4. One important aspect of outcome is time to full weight bearing. The authors had good results in terms that all patients were walking full weight bearing at the time of discharge; however, exact time would be of much more interest.

5. As a number of patients in both groups had periprosthetic fractures, knee range of movement (ROM) will depend upon the pre-injury ROM with the knee replacement in situ. This issue also needs clarification.

Conflict of interest No funds were received in support of this study. 